Clinical Proteomics Journal

Copyright (CHumana Press Inc.

All rights of any nature whatsoever are reserved.

ISSN 1542-6416/04/01:45-67/\$25.00

\title{
Molecular Targets
}

\section{New Targets for an Old Drug}

\section{A Chemical Proteomics Approach to Unraveling the Molecular Mechanism of Action of Methotrexate}

\author{
Leticia M. Toledo-Sherman, ${ }^{* \prime}$ Leroi Desouza, ${ }^{\dagger}$ Christopher M. Hosfield, ${ }^{\dagger}$ \\ Linda Liao, Kelly Boutillier, Paul Taylor, Shane Climie, \\ Linda McBroom-Cerajewski, and Michael F. Moran*,
}

'MDS Proteomics Inc., 25I Attwell Drive, Toronto, ON M9W 7H4, Canada

\begin{abstract}
Methotrexate has been a clinical agent used in cancer, immunosuppression, rheumatoid arthritis and other highly proliferative diseases for many years, yet its underlying molecular mechanism of action in these therapeutic areas is still unclear. We present a chemical proteomics approach that uses ultra-sensitive mass spectrometry coupled to an inverse protein-ligand docking computational technique to unravel the mechanism of action of this drug. Using methotrexate tethered to a solid support we were able to isolate a significant number of proteins. We effectively captured a large portion of the de novo purine metabolome and propose a
\end{abstract}

pathway architecture similar to that seen in signaling pathways and consistent with substrate channeling. More importantly, we were able to capture protein targets that could potentially provide new insights into the mechanism of action of methotrexate in rheumatoid arthritis and immunosuppression. The application of this approach to other drugs and drug candidates may facilitate the prediction of unknown and secondary therapeutic target proteins and those related to the side effects and toxicity. These results demonstrate that this proteomics technology could play an important role in drug discovery and development since it allows monitoring of the interactions between a drug and the protein content of a cell.

*Author to whom all correspondence and reprint requests should be addressed:

E-mail: 1toledo@mdsp.com, mmoran@utoronto.ca

${ }^{+}$Authors contributed equally to this work. 
Key Words: Methotrexate; targets; mechanism of action; chemical proteomics; pharmaco-proteomics; metabolome; inverse docking; substrate channeling.

\section{Introduction}

One of the most expensive, yet important aspects of drug discovery and development is the clinical evaluation of emerging therapeutics. Yet, it is at this stage that most drug candidates fail to show efficacy or tolerable toxicities and are withdrawn (1). Furthermore, a large number of drugs already in clinical use today have unknown or unclear molecular mechanisms of action and toxicity. Precise knowledge of the mechanism of action of these drugs would facilitate critical decisions to be made regarding label and patent expansion, as well as the development of secondgeneration therapeutics. A promising aspect of the emerging field of proteomics is the development of sensitive tools and methods for facilitating an understanding of key interactions between a drug and its targets at the molecular level (2), thus allowing a better decision-making process so that only compounds with a high probability of showing the required efficacy profile and low toxicity are considered for the clinic.

Already, chemical proteomics, the capturing of a select proteome with a chemical agent or drug, has shown potential in accelerating drug discovery and development $(3,4)$. Recently, a chemical proteomics approach was used to identify the targets of the widely used quinolines and offered an insight into the mechanism of action of these drugs (5). We are interested in developing such methods and have access to mass spectrometry instrumentation with ultra-sensitivity (6). One of these methods is a pharmaco-proteomics approach to understanding the interaction between a drug and its targets. We have developed an affinity-based chemical proteomics platform that uses small molecules (e.g., a drugs or drug candidates) attached to solid supports in such a way that when exposed to a biological sample its interacting protein partners can be captured and identified. Captured proteins are isolated by gel electrophoresis, readily analyzed by mass spectroscopy and identified by searches against sequence databases. With this technique, we are able to identify proteins that are interacting directly with the drug probe or interacting indirectly via protein-protein engagement with direct interactors. For the classification of direct vs indirect interactions we have coupled our experimental methods with a computational technique termed inverse docking. This procedure is used to dock a ligand against a library of protein structures. Recently, this inverse docking procedure was shown to be effective in identifying potential new targets of $4 \mathrm{H}$ tamoxifen and vitamin E (7), respectively. This tool has also been used to identify potential toxicity and side effect-related protein targets of a small molecule (8). With the advent of structural genomics initiatives and given the richness of protein structures already present (9) in the protein database (http://www.rcsb. org $/ \mathrm{pdb}$ ), we expect that the three dimensional structures of many of the protein identified in our experiments would be readily accessible or predictable by homology modeling from related protein structures. Proteins for which inverse docking proposes binding modes representing low energy complexes, as scored by a consensus scoring technique (10) combining five different score functions, are deemed hits and worth considering as direct binders of the ligand.

To demonstrate the potential of combining these two technologies, we selected the widely used drug methotrexate (MTX). Methotrexate is a folate antimetabolite that has been used extensively for the treatment of highly proliferative diseases such as rapidly growing tumors, acute leukemia, rheumatoid arthritis, psoriasis, pneumocystis carinii caused-pneumonia associated 
with AIDS, and other chronic inflammatory disorders. Methotrexate has recognized efficacy as an anti-inflammatory (11), immunosuppressive (12) and anticancer agent (13). In inflammation and immunosuppression, despite its wide use and several theories behind its efficacy, the underlying molecular mechanism of action of MTX remains unclear. In cancer, the mechanism of action of MTX has been understood as occurring because of cytotoxicity originating from the accumulation of the corresponding polyglutamated MTX metabolites in cells (14). Methotrexate is taken into cells by reduced folate carrier (RFC) protein, where it is polyglutamated by folylpolyglutamate synthetase (FPGS). Upon polyglutamation, MTX binds to dihydrofolate reductase (DHFR) interrupting the conversion of dihydrofolate to the activated N5,N10methylene-tetrahydrofolate. N5,N10-methylenetetrahydrofolate is the main methylene donor in de novo purine biosynthesis, it provides the methyl group in the conversion of deoxyuridine-5'-monophosphate (dUMP) to deoxythymidine-5'-monophosphate (dTMP) for DNA synthesis and for many transmethylation processes. The polyglutamated metabolite is subject to back glutamyl hydrolysis by $\gamma-\mathrm{GH}$ and efflux from cells.

The three main targets of antifolate drugs in the clinic are DHFR, thymidylate synthase (TS), and glycinamide ribonucleotide transformylase (GART) (15). Several newer generation classical (polyglutamates) and nonclassical (nonpolyglutamates) antifolate drugs are now under clinical evaluation with promising results (16). It is well known now that MTX and other antifolates inhibit other proteins besides DHFR, TS, and GART. Amino-imidazolecarboxamide-ribonucleotide transformylase (AICARFT) (17), serine hydroxymethyltransferase (SHMT) (18), FPGS (19), $\gamma-\mathrm{GH}$ (20), and reduced folate carrier (RFC) (21) are known to bind antifolates.
The main problem with classical antifolates is that accumulation of polyglutamated metabolites causes drug resistance in cells. Several mechanisms of resistance (22) defective transport through cell membranes, amplification of DHFR, reduced expression of FPGS and upregulation of $\gamma-\mathrm{GH}$ have been identified as the underlying basis for the development of resistance to antifolates. Because of the increased resistance with current antifolate drugs, there is a need for new antifolate targets. The development of clinical diagnostic markers for antifolate drug resistant tumors would also be beneficial in deciding which therapies to choose for those tumors. Of equal importance to clinical applications, is the understanding of the molecular mechanism of action and toxicity of existing and emerging antifolates therapeutics.

In order to address some of these issues we set out to investigate the underlying molecular mechanism of action of MTX. Structurally, the MTX-DHFR (23) (Fig. 1A) pair is one the best understood ligand-protein systems and at the time of writing a search of the protein databank for the keyword MTX resulted in 66 entries. Most of these entries are of MTX or derivatives in complexes with DHFR from different species and DHFR mutants, but structures for TS $(1 \mathrm{AXW})(24)$ also exist. The crystal structure of GART in complex with a molecule of glycinamideribonucleotide (GAR) and a folate analog is also available (1CDE) (25). In all these structures the aminopterin and the $\alpha$-carboxylate groups of the molecule are buried inside the binding site and make key hydrogen bond interactions with the protein, while the $\gamma$-carboxylate group protrudes out of the cavity (Fig. 2). For our studies, we used the readily commercially available MTX agarose reagent. This material is a mixture resulting from linkages to the support through the $\alpha$ - and $\gamma$-carboxylates of the molecule. According to the structures of MTX com- 
plexes, we would expect that only the material linked through the $\gamma$-carboxylate should be productive in binding proteins from a cell lysate, as the linkage through the $\alpha$-carboxylate would be stericly hindering if we assume a similar binding conformation is required with other proteins.

\section{Methods}

\section{Preparation of Cell Lysates}

HEK 293 cells (typically 107) were harvested, washed with PBS, then lysed in a buffer containing $20 \mathrm{mM}$ Tris, $150 \mathrm{mM} \mathrm{NaCl}, 1 \% \mathrm{NP}-40$, $0.5 \%$ sodium deoxycholate supplemented with protease inhibitors. After incubation for $30 \mathrm{~min}$ at $4^{\circ} \mathrm{C}$ with shaking, the lysates were clarified by centrifugation $(27,000 \mathrm{~g})$. In some experiments, cells were lysed using 20 strokes of a Dounce homogenizer in the absence of detergents, although since similar results were obtained, detergent-based lysis was most-often used. In most cases, proteins in the clarified lysate were directly applied to MTX-affinity columns (see section on Affinity Chromatography). While optimizing the protocol, however, several experimental variations were tested on cell lysates including concentration by ammonium sulfate precipitation, or removal of nucleic acid with Streptomycin sulfate. In such cases, the protein sample was desalted using a PD 10 protein-desalting column (Pharmacia), which had been pre-equilibrated in the same buffer (10 $\mathrm{mM}$ potassium phosphate $\mathrm{pH}$ 7.5).

\section{Affinity Chromatography}

The desalted lysate was loaded onto a column of pre-equilibrated MTX-agarose (Sigma, $50 \mu \mathrm{L}$ bed volume) or sepharose 4B agarose as a negative control. The lysates was allowed to slowly flow through the matrix under gravity flow. The columns were then washed with $4 \times 0.6 \mathrm{~mL}$ of the same potassium phosphate buffer with various concentrations of $\mathrm{NaCl}$ (usually $0.4 \mathrm{M}$ but occasionally $1.0 \mathrm{M}$ ), followed by a quick rinse with $0.2 \mathrm{~mL}$ potassium phosphate, $0.1 \mathrm{M}, \mathrm{pH} 6.0 ; 100 \mathrm{mM} \mathrm{NaCl}$ and eluted with $2 \times 100 \mu \mathrm{L}$ of $10 \mathrm{mM}$ MTX in potassium phosphate, $0.1 \mathrm{M}, \mathrm{pH} 5.6$; and $100 \mathrm{mM} \mathrm{NaCl}$. Eluates containing the proteins eluted by MTX were then concentrated by spinning through microcon 3 (from Amicon). Retentates from the microcons were then loaded onto SDS-PAGE 4-15\% gradient mini gels (Bio-Rad). Gels were stained with Gel Code Blue (Pierce), de-stained and imaged. Bands of interest were excised, diced, trypsin digested and sent for mass spectrometry analysis.

\section{Protein Identification by Mass Spectrometry}

Tryptic peptides recovered from individual gel bands were separated by reverse phase chromatography on C18 resin and directly injected into a mass spectrometer with an automated sample loading device from 96 well plates. Two types of mass spectrometry platforms were used: (1) quadrupole ion traps (LCQ Deca, Thermo Finnigan), and (2) customized quadrupole time-of-flight hybrid instruments (QSTAR Pulsar, MDS Sciex). Both instrument types were operated in datadependent mode, which produces tandem MS spectra of all peptide species present above a programmed threshold. The spectra generated were analyzed on a custom-built multi-node server platform (RADARS, ProteoMetrics), which uses two database searching programs, Sonar (ProteoMetrics) and Mascot (Matrix Sciences). The identities of the proteins were obtained from database queries of the MS derived data. The databases searched included NCBI non-redundant protein, EMBL ensemble predicted protein, NCBI human chromosomal, and proprietary internal databases.

\section{Docking Studies}

Protein X-ray crystal structure coordinates were downloaded from the protein data bank. The corresponding pdb codes for the proteins 


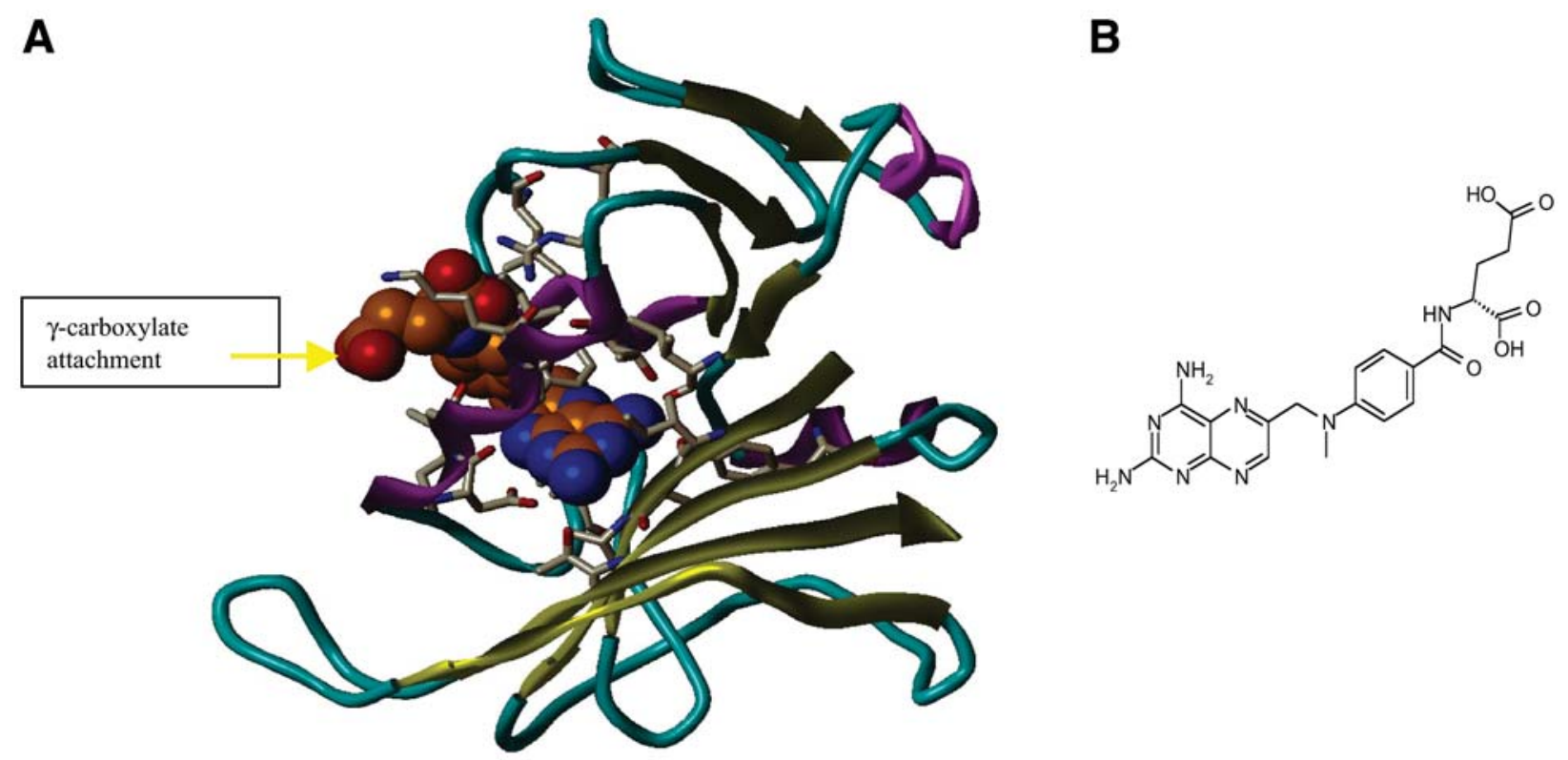

Fig. I. (A) Crystal structure of Methotrexate complexed within the active site of dihydrofolate reductase showing the $\gamma$-carboxylate protruding out of the cavity. (B) Methotrexate molecule.
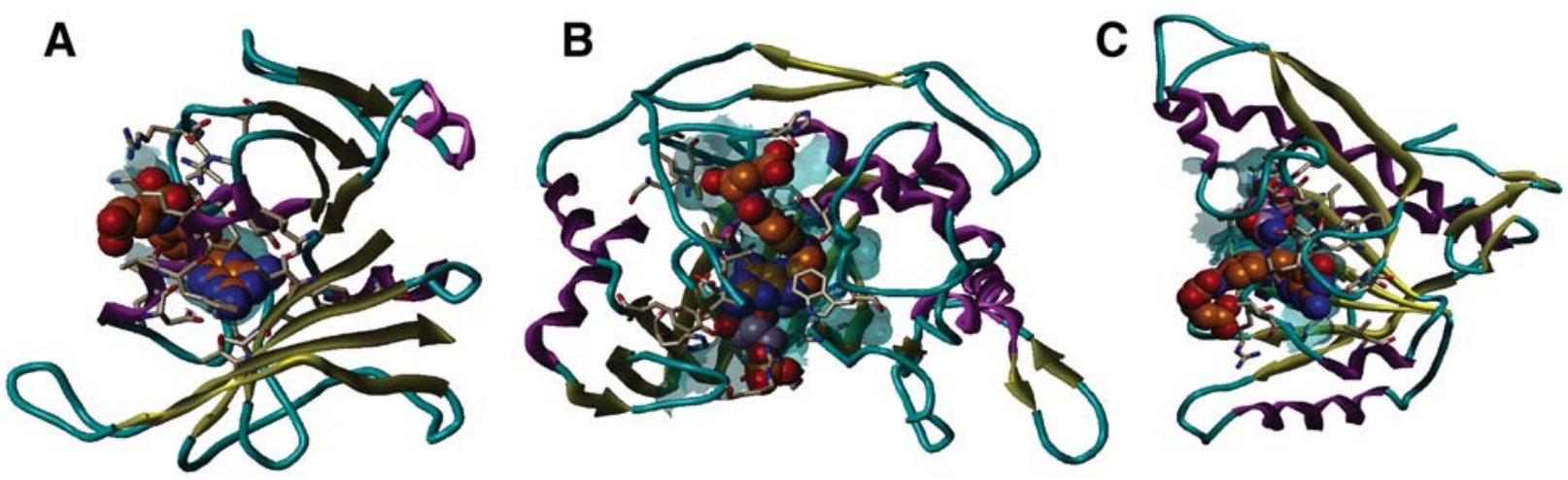

Fig. 2. Crystal structure of (A) MTX-DHFR (IRG7), (B) MTX-TS (IAXW), and (C) folate-GART (I CDE), respectively showing $\gamma$-carboxylate of methotrexate or folate derivative protruding out of the binding cavities of all three enzymes.

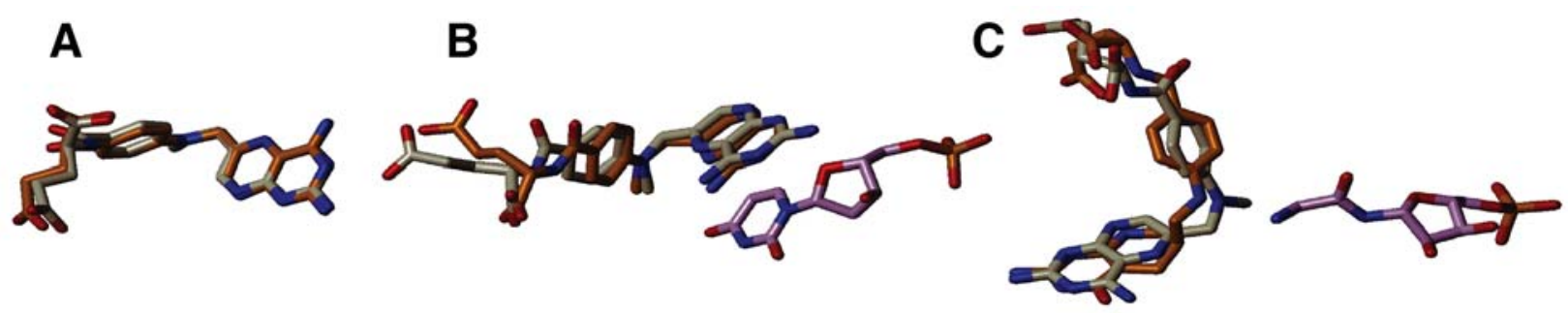

Fig. 5. Overlap of docking poses (white) for methotrexate over the experimentally observed positions (gold) for all proteins. RMS (A) deviations were (A) 0.4 I for mtx-DHFR (IRG7), (B.) 1.07 for mtx-TS-DUMP (IAXW), and (C) 0.82 for folate-GART (I CDE), respectively. 
Table 1

Proteins Identified by Mass Spectrometry ${ }^{a}$

\begin{tabular}{|c|c|c|c|}
\hline Protein Identified & $\begin{array}{l}\text { Known } \\
\text { folate } \\
\text { targets }\end{array}$ & $\begin{array}{c}\text { New proposed } \\
\text { targets of } \\
\text { methotrexate }\end{array}$ & PDB codes \\
\hline Dihydrofolate reductase (DHFR) & $\sqrt{ }$ & & 1RG7 \\
\hline Thymidylate Synthetase (TS) & $\sqrt{ }$ & & $1 \mathrm{AXW}$ \\
\hline Glycinamideribonucleotide transformylase (GART) & $\sqrt{ }$ & & $1 \mathrm{CDE}$ \\
\hline Aminoimidazole ribonucleotide synthetase (AIRS) & & & 1CLI \\
\hline Glycinamideribonucleotide synthase (GARS) & & & $1 \mathrm{GSO}$ \\
\hline Amidophosphoribosyltransferase & & $\sqrt{ }$ & $1 \mathrm{AO} 0$ \\
\hline AIR carboxylase & & & 1D7A \\
\hline SAICAR synthetase & & & $1 \mathrm{~A} 48$ \\
\hline Hypoxanthine phosphoribosyltransferase (HPRT) & & $\sqrt{ }$ & $1 \mathrm{D} 6 \mathrm{~N}$ \\
\hline Deoxycytidine kinase & & $\sqrt{ }$ & $1 \mathrm{P} 62$ \\
\hline Deoxyguanosine kinase & & $\sqrt{ }$ & 1JAG \\
\hline Pyridoxal kinase & & $\sqrt{ }$ & 1LHR \\
\hline Glutamate-ammonia ligase (glutamine synthase) & & & 1F52 \\
\hline Inosine monophosphate dehydrogenase (IMPDH) & & $\sqrt{ }$ & 1ME9 \\
\hline Pterin-4- $\alpha$-carbinolamine dehydratase (PCD) & & $\sqrt{ }$ & $1 \mathrm{DCP}$ \\
\hline Nudix 1 & & & 1G9Q \\
\hline Nudix 5 & & $\sqrt{ }$ & $1 \mathrm{KHZ}$ \\
\hline Divalent Cation tolerant protein CUTA & & & 1P1L \\
\hline Glutathione synthase & & & 1GSA \\
\hline Glycogen phosphorylase & & $\sqrt{ }$ & $1 G G N$ \\
\hline Propionyl CoA carboxylase & & & Unknown \\
\hline
\end{tabular}

${ }^{a}$ Check denotes new methotrexate targets.

used for the docking study are given in Table 1. All waters of crystallization were removed and all hydrogen atoms were added to the proteins. Kollman-All charges were used for all protein atoms using SYBYL (Tripos, St. Louis, MO) and the protein file saved as a Sybyl mol2 file. The initial conformation of the MTX was extracted from the crystal structure complex of dihydrofolate reductase and MTX (1RG7). Coordinates for the molecule were extracted, all atom types checked and corrected, all hydrogens were added and GasteigerHuckel charges were applied. For the inverse docking procedure, the MTX molecule was docked into the active sites of all proteins listed in Table 1 using the standard default settings of the program GOLD (CCDC, Cambridge, UK). The resulting binding modes were visually inspected for poses where the $\gamma$-carboxylate of MTX protruded out of the binding site as observed for DHFR and could be considered compatible with binding. Scoring of the poses was performed using the consensus-scoring module CSCORE (Tripos, St. Louis, MO).

\section{Results}

Conceptually, proteins may associate with the immobilized ligand either through a direct binding interaction or by an association with a direct binding protein. The efficiency of the interaction, as reflected in the amount of a pro- 


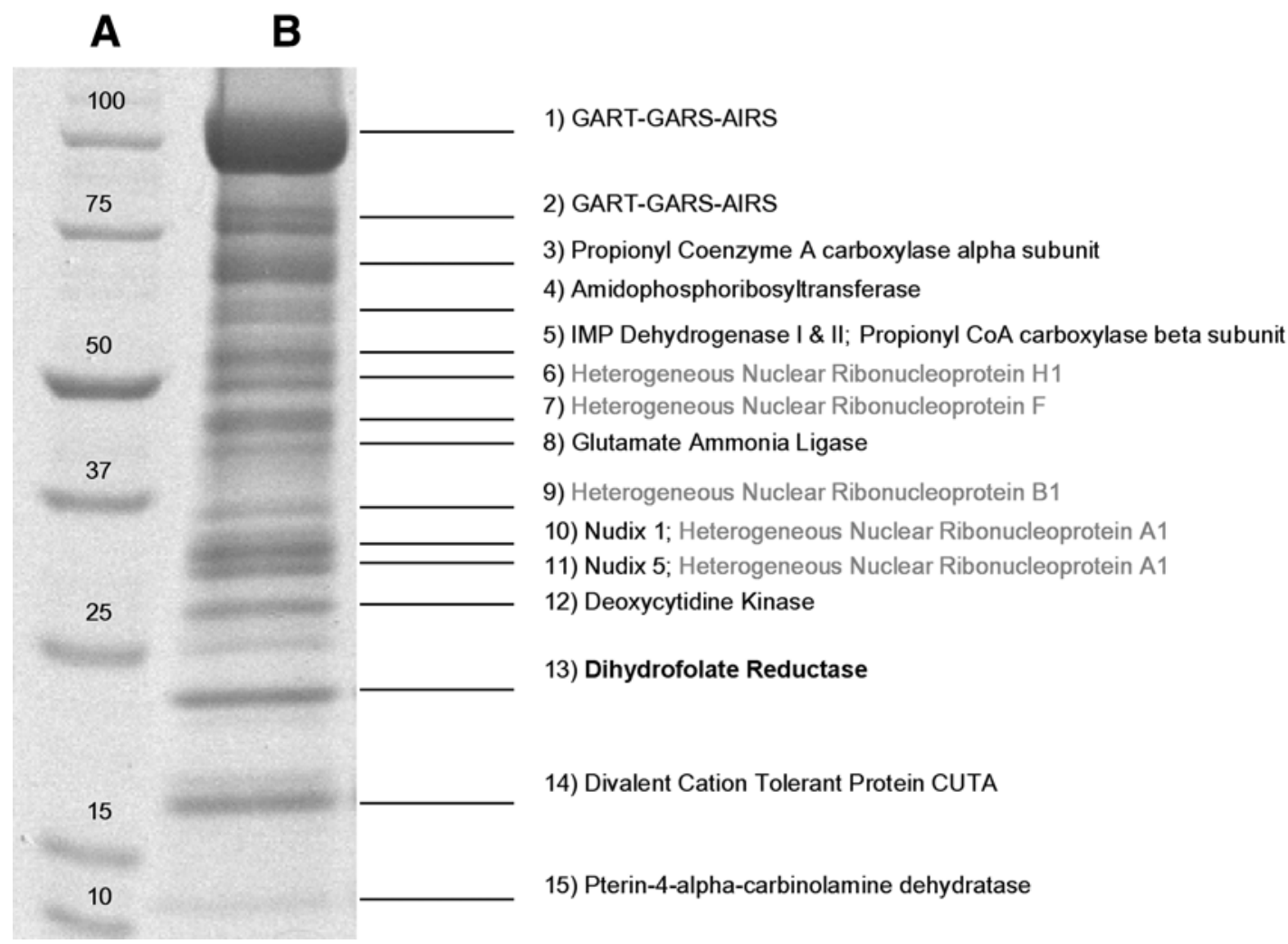

Fig. 3. Protein signature or fingerprint of methotrexate probe. Lane $A$ : molecular weight markers $(\mathrm{kDa})$, lane $B$ : bands corresponding to proteins identified by mass spectrometry analysis.

tein recovered, is a function of several parameters including both the abundance of the protein, its affinity for the MTX-loaded resin, and the chromatographic and buffer conditions employed. As expected, initial affinity-capture experiments for MTX-interacting proteins resulted in the isolation of a band on SDSPAGE that was identified by MS as DHFR. DHFR was used as an internal control according to which chromatographic conditions were optimized for subsequent experiments.

After optimal binding and elution conditions were established, we were able to reproducibly isolate a significant number of proteins that associated with the immobilized MTX probe, as indicated in the Coomassiestained gel in Fig. 3. The identity of the isolated proteins was determined by MS analysis, and the results are listed in Table 1 along with the corresponding PDB code.

In addition to DHFR, a second known MTX target, GART, was identified. Many of the other proteins identified, such as amidophosphoribosyltransferase, phosphoribosylaminoimidazole (AIR) carboxylase, glycinamide ribonucleotide synthase (GARS), aminoimidazole ribonucleotide synthetase (AIRS), phosphoribosylaminoimidazolesuccinocarboxamide (SAICAR) synthase, hypoxanthine amidophosphoribosyltransferase (HGPRT) and inosine monophosphate dihydrolase (IMPDH) are enzymes involved in either the de novo or salvage purine synthesis pathways (Fig. 4). Interestingly, we also identified glutamate ammonia ligase, an enzyme involved in the production of glutamine, a consumable used in nucleotide 


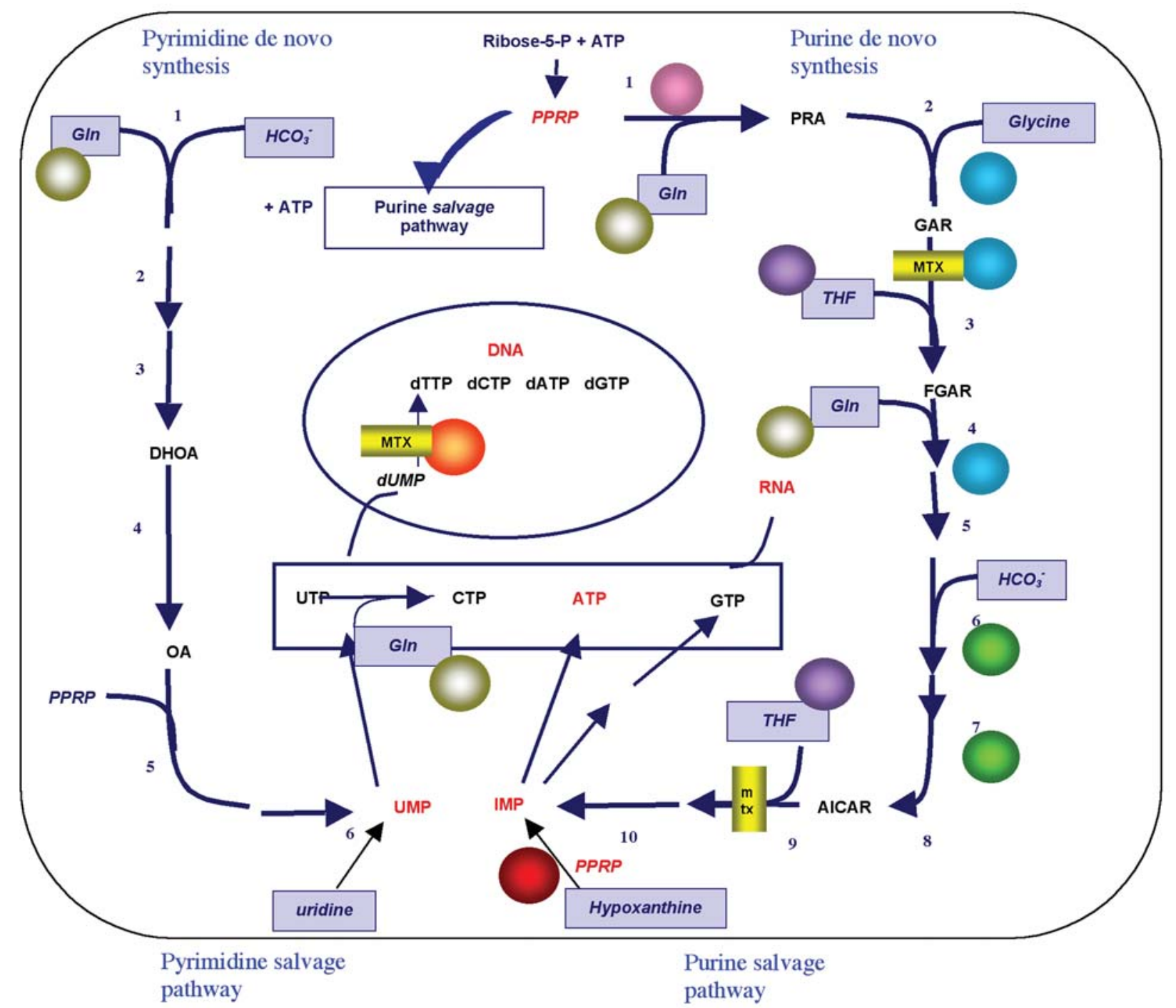

Fig. 4. Diagram of purine and pyrimidine de novo and salvages pathways showing enzymes that have been isolated by the methotrexate probe. Colored spheres represent different enzymes. Glutamine ammonia ligase (grey), Amido phosphoribosyltransferase (lilac), GARS-AIRS-GART (cyan), DHFR (purple), SAICAR synthaseAIR carboxylase (green), HPRT (red), and TS (orange). Empty circles represent enzymes in this pathway that were not found, FGARAT, succino adenylo-succinate lyase, and AICART-IMP synthase.

synthesis. Deoxycytidine kinase and deoxyguanosine kinase are also involved in DNA synthesis. Other proteins also consistently found were Pterin-4- $\alpha$-carbinolamine dehydratase (PCD), nudix 1, and nudix 5, CUTA, pyridoxal kinase, glycogen phosphorylase and glutathione synthase. Although not seen in initial experiments, we were able to identify TS (a known MTX-interactor) after protocols were adjusted to include $10 \mathrm{mM}$ dUMP in the binding conditions. Finally, it should be noted that several "background" proteins including het- 
erogeneous nuclear ribonucleoproteins, actin, tubulin, and heat shock proteins were also identified in these experiments. These highly abundant proteins are routinely identified by our platform using agarose-based isolation techniques and are not considered to be interacting directly with the immobilized MTX.

\section{Protein-MTX Docking}

As expected, crystal structures for all but one of the proteins captured in our experiments were available from the protein data bank. Similarly, all the crystal structures for the proteins known to bind MTX that were not identified in our experiments, except for RFC protein, were available. As previously mentioned, the crystal structures of DHFR, TS, and GART (Fig. 2) as complexes with MTX or folates were available. Visual inspection of these structures indicated that in all the structures the $\gamma$-carboxylate group protrudes out of the active site cavity and is consistent with affinity capture by our MTX probe. These represented great tools for verifying the predictability of the docking exercise. If the docking exercise could reproduce the binding modes experimentally observed in the X-ray crystal structures of these three protein complexes, then it is realistic to expect that docking runs on other proteins would produce reasonable solutions as well, and would predict direct vs indirect binding. We performed inverse docking of MTX or the folate molecule into the binding site of the three proteins and investigated the ten best docking poses for each.

We found that in all three cases several poses of the MTX or folate molecule approximated the experimentally observed positions with a high degree of accuracy. The pose with the greatest overlap over the experimentally observed position was taken as correct and the root mean square (RMS) deviation from the experimentally observed positions measured. RMS deviations ( $\mathrm{A})$ were: 0.41 for MTX-DHFR
(1RG7), 1.07 for MTX-TS (1AXW), and 0.82 for folate-GART (1CDE), respectively. Figure 5 shows the overlap between the acceptable poses and the experimental positions for all three proteins. This validation exercise indicated that docking could indeed be a useful tool in rationalizing the type of binding interactions responsible for the recovery of the MTX-associated proteins. In all cases in which a crystal structure was available for the recovered proteins, visual inspection of the structure followed by protein ligand docking was performed. Proteins for which proposed binding modes represent low energy complexes, compatible with the affinity ligand binding, are deemed direct binders or targets of MTX. Table 1 lists all the proteins for which docking results predicted direct interactions and for which evidence of MTX or folate binding was present in the literature.

\section{Discussion}

\section{Known Targets of MTX}

Figure 4 shows a schematic representation of the nucleotide de novo and salvage pathways highlighting the proteins (colored spheres) that were identified in our experiments. Remarkably, a great number of enzymes involved in these pathways including several enzymes that are not directly dependent of folate cofactors were captured. In essence this amounted to capturing a metabolome. This suggests that this metabolic pathway is effectively scaffolded together through protein-protein interactions, possibly as a means to facilitate forms of co-regulation of the constituent enzymes and achieve a more efficient anabolic process, as described below (see section on Enzyme Channeling). This is consistent with paradigms in both signal transduction pathways, and pathways for macromolecular biosynthesis, such as DNA replication and transcription. 
As expected, DHFR was identified as a strongly staining band in the gel. The interaction between MTX and DHFR has been measured at approx $10 \mathrm{nM}$ (26). Addition of $10 \mathrm{mM}$ dUMP to the medium facilitated the recovery of another MTX target, TS. TS catalyses the reductive methylation of dUMP to dTMP, which is later phosphorylated to dTTP for incorporation into DNA (27). This is a key step in DNA synthesis and the only pathway to dTMP. This protein is a major target of several anticancer agents such as the widely used dUMP derivative anticancer agent 5-flourouracil (FU). The rationale for dUMP stimulating the association of TS with the MTX matrix is that in the conversion of dUMP to dTMP, in order for the methyl group transfer to occur from an activated folate molecule to dUMP, a complex needs to be formed in which the molecular planes of folate and dUMP are superposed via $\pi-\pi$ stacking interactions. Therefore the efficiency of MTX binding to TS is dependent on a $\pi-\pi$ interactions with dUMP. Consistent with this structural observation and our results, the $\mathrm{Kd}$ for the interaction of MTX with TS in the presence of dUMP has been measured at $24.5 \mu \mathrm{M}$, but in the absence of dUMP there is no detectable interaction (28).

The association of GART with the MTX matrix was not surprising since it is one of two folate dependent enzymes in the de novo purine synthesis. This enzyme catalyses the transfer of a formyl group from 10-formyltetrahydrofolate to the amino group of glycinamide ribonucleotide (GAR). Hence, we postulate this association is the consequence of a direct interaction between GART and the MTX ligand. Over the last decade or so, GART has become and important target for anticancer therapy.

\section{New Proposed Targets of MTX}

Besides DHFR, TS and GART, several new proteins were identified that our inverse dock- ing experiments suggest are new targets of MTX. For most of these proteins, there is also evidence in the literature for binding by folates, MTX or MTX-derivatives, or by chemotypes that can make similar hydrogen bonding interactions as the pterin group of MTX. The corresponding protein-MTX complexes proposed by our inverse-docking procedure are presented in Figure 6.

\section{Amido Phosphoribosyltransferase}

Amido phosphoribosyltransferase catalyses the committed step in purine biosynthesis. This enzyme catalyses the addition of an amine group to phosphoribosylpyrohosphate (PPRP). This transformation takes place as two separate reactions at two distinct active sites in this enzyme. The enzyme contains glutaminase activity in the $\mathrm{N}$-terminal domain and phosphoribosyl transferase in the C-terminal domain. PPRP binds at the phosphoribosyltransferase site and causes a conformational change in the enzyme allowing glutamine to bind at the glutaminase site (29). The ammonia generated at the glutaminase site is rapidly channeled through the formation of a hydrophobic tunnel to the PPRP molecule positioned for nucleophilic attack by the incoming NH3. The resulting phosphoribosylamine (PRA) is a highly unstable intermediate under physiological conditions and is rapidly transferred to the next enzyme in the pathway, GARS, where it is converted to GAR.

Amido phosphoribosyltransferase is subject to feedback inhibition by end products of the pathway AMP, GMP, and IMP through interaction at two allosteric binding sites with differing preference for AMP at one site and GMP and IMP at the other. We performed visual inspection followed by docking MTX at both sites. Our docking experiments resulted in several binding modes of MTX that are consistent with binding at the GMP allosteric binding site (1AO0) (30), but not at the AMP 

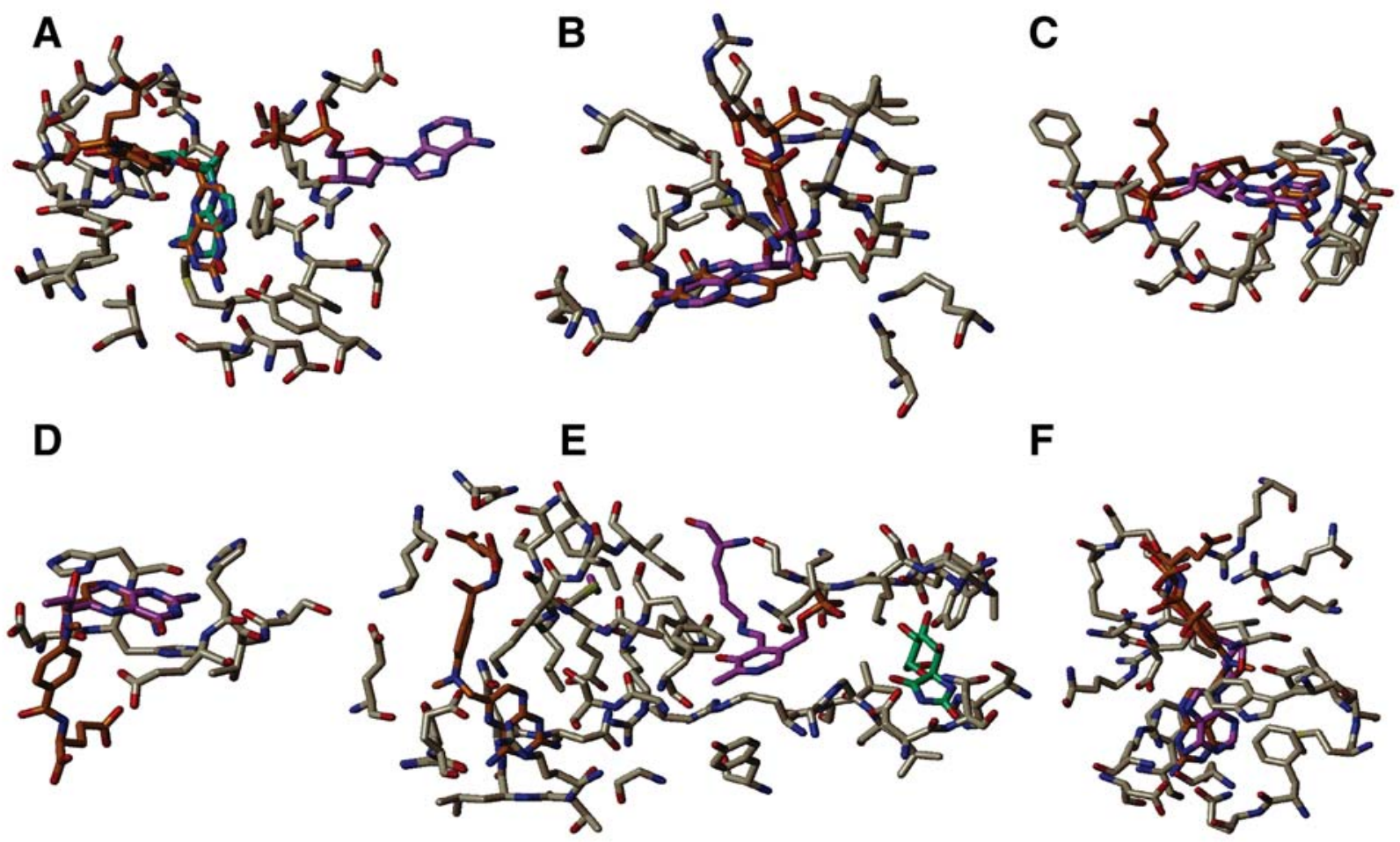

$\mathbf{F}$
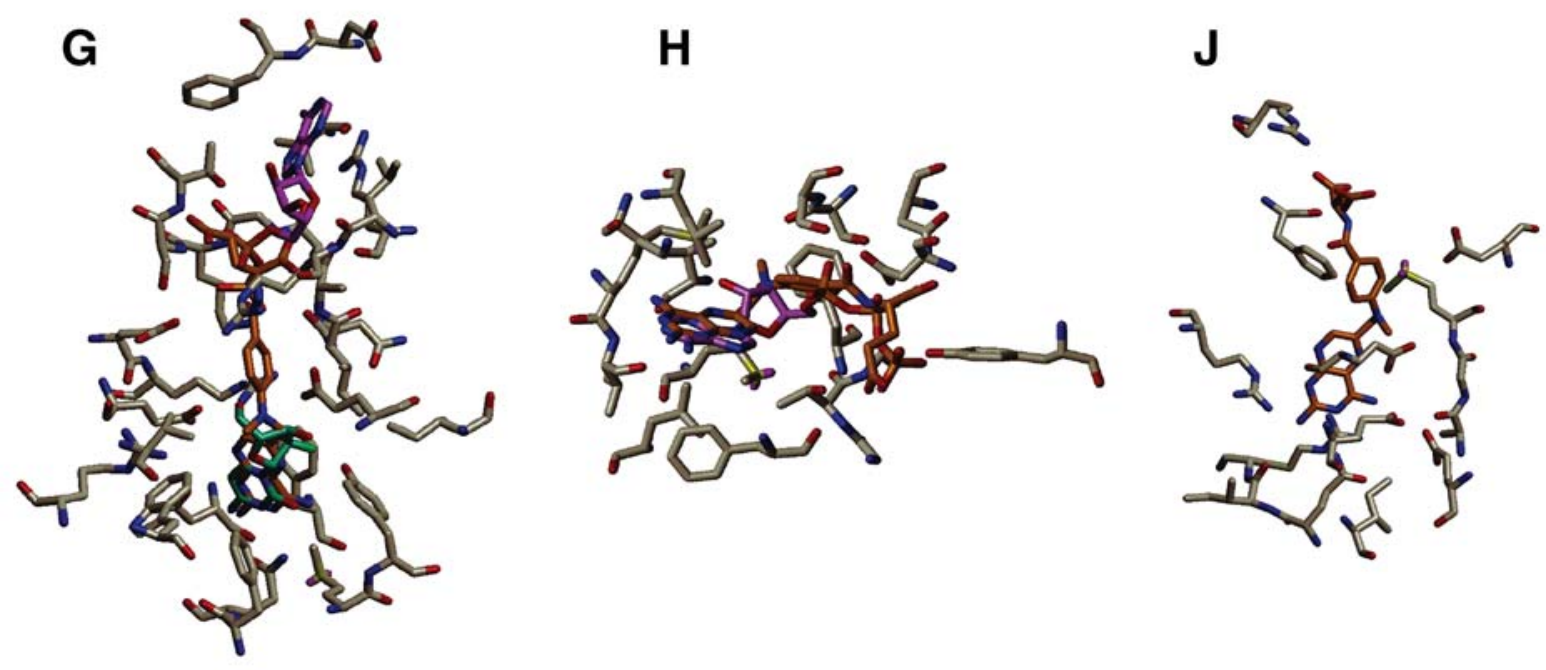

Fig. 6. Proposed binding modes for methotrexate complexes with proteins postulated to represent new targets of this drug (MTX is in orange and when applicable is superposed over the experimentally complexed ligand from the X-ray structure. Only active site residues shown and some residues have been omitted for clarity). (A) Amido phosphoribosyltransferase-ADP (lilac), GMP (light green); (B) IMPDH-IMP (lilac); (C) HGPRTGMP (lilac); (D) PCD-7,8-dihydrobiopterin (lilac); (E) glycogen phosphorylase-glucopyranose spirohydantoin (light green), pyridoxal phosphate (lilac); (F) deoxyguanosine kinase-ATP (lilac); (G) deoxycytidine kinase-ADP (lilac), prodrug AraC (light green); (H) pyridoxal kinase-ATP (lilac); and (J) Nudix 5. 
site. Our results are corroborated by evidence in the literature that MTX inhibition of purine de novo synthesis in leukemia cells occurs before the folate dependent steps carried out by GART and AICARFT. On treatment with MTX the de novo pathway is completely blocked, accumulation of GAR and AIRCAR intermediates are minimal, whereas accumulation of 5-phosphoribosyl-1-pyrophosphate is three- to four-fold (31). This is consistent with the interpretation that the enzyme being inhibited by MTX is amido phosphoribosyltransferase. Further in vitro assays performed with MTX-Glu ${ }_{5}$, the active metabolite of MTX in cells also showed that amido phosphoribosyltransferase is inhibited (32).

A widely accepted mechanism of action for low dose MTX in rheumatoid arthritis (RA) is based on the theory that MTX mediates adenosine release. This has been considered secondary to the inhibition of AICARFT and accumulation of AICAR. More recently it has been shown that in addition to adenosine release, MTX induces apoptosis of activated T-cells in the S/G2 phase of the cell cycle (33). A more recent study, in mitogen stimulated T-lymphocytes, showed by radiolabeling studies of reaction intermediates that MTX blocks the committed step in purine synthesis. The authors postulate that direct inhibition of amidophosphoribosyltransferase could be the underlying mechanism for the efficacy of MTX in RA (34). The fact that we consistently isolated this enzyme under a variety of conditions provides strong evidence of its direct inhibition by MTX. This finding is of paramount importance. If the inhibition of amido phosphoribosyltransferase by MTX is indeed responsible for the efficacy of this drug in RA, this could present the pharmaceutical industry with a new RA target and open wide opportunities for the search of new drug chemotypes that may be less prone to resistance than MTX. Structure-based design would be highly amenable because of the knowledge of the three dimensional structure of this enzyme.

\section{Inosine Monophosphate Dehydrogenase (IMPDH)}

Our experiments identified both isoforms IMPDH1 and IMPDH2. IMPDH catalyses the nicotinamide adenosine dinucleotide dependent conversion of inosine 5'-phosphate to xanthosine $5^{\prime}$ phosphase, the first step in the de novo synthesis of guanine nucleotides. Rapid proliferating cells such as lymphocytes depend on the availability of nucleotide pools. It is known that the activity of IMPDH is higher in rapid proliferating cells. Because of these cell requirements, IMPDH has become and important target for immunosuppressive, anticancer and antiviral therapies. Several IMPDH inhibitors are now being evaluated in the clinic (35). Since this enzyme binds the inosine moiety, and other enzymes that bind IMP have been known to also bind folate analogs, we postulate that MTX could bind this enzyme directly. Docking MTX in the recently solved crystal structure of the IMPDH1 in complex with IMP (1ME9) (36) generated several binding modes compatible with direct binding to our affinity column. In several poses the MTX molecule binds at the IMP/XMP site of the protein with the pterin group overlapping the position typically occupied by the head group of the IMP/XMP molecules and make similar hydrogen bond interactions. The benzyl and glutamate groups sit over the sugar and phosphate of IMP / XMP and point towards the opening of the cavity. The crystal structure of IMPDH with the immunosuppressive inhibitor micophenolic acid has revealed the mode of inhibition of this agent. Micophenolic acid occupies the NAD site of IMPDH and acts by blocking the conversion of IMP to XMP (37). It is possible that MTX and micophenolic acid both 
derive their immunosuppressive activities by inhibiting IMPDH but through slightly different molecular mechanisms. A provocative possibility for the efficacy of MTX as an immunosuppressive agent is that this effect may be caused at least in part through direct inhibition of IMPDH.

\section{Hypoxanthine-Guanine Phosphoribosyltransferase (HGPRT)}

Hypoxanthine-guanine phosphoribosyltransferase is the most important enzyme of the salvage pathway. This enzyme catalyses the salvage conversion of hypoxanthine and guanine to IMP and GMP, respectively, by facilitating the addition of the bases to the activated PPRP molecule (38). This enzyme, like amidophosphoribosyltransferase, is involved in amine addition to the PPRP. The activity of salvage enzymes like HGPRT is higher than the activity of enzymes involved in the de novo pathways. It has been puzzling that agents such as MTX, believe to act primarily on de novo enzymes, are effective in spite of the presence of highly active salvage enzymes. This has recently been accounted for, at least in part, by new observations showing that MTX can reduce the activity of HGPRT (39). Other observations suggest in vivo inhibition of HPRT by MTX. For example, deficiency in HGPRT is known to result in higher levels of PRPP and an acceleration of purine biosynthesis by the de novo pathway (40). Treatment with MTX also produces an increase on levels of PRPP (41) and this effect is reversible upon treatment with hypoxanthine. Our docking experiments are also consistent with direct binding as MTX can fit in the binding pocket of HGPRT (1D6N) (42) with good overlap over the positions occupied by hypoxanthine monophosphate with the glutamate group of MTX protruding out of the cavity. The evidence in the literature and our findings indicate direct inhibition of HGPRT by MTX. This could contribute, at least in part, to the efficacy of MTX as an anti-cancer agent.

\section{Pterin-4- $\alpha$-Carbinolamine Dehydratase (PCD)}

PCD catalyses the dehydration of $4 \alpha-$ hydrozytetrahydrobiopterins to the corresponding dihydropterins. Dihydrobiopterin is a substrate of pteridine reductase, an enzyme known to bind MTX directly (43). Here we speculate that because both enzymes bind the same dihydrobiopterin, PCD is binding directly to the MTX probe. Our docking experiments on the structure of PCD from the crystallographic complex with biopterin (1DCP) (44) supports direct binding. In our docking experiments, several docking poses were found where the pterin moiety of MTX exactly overlaps over the biopterin molecule in the complex and the $\gamma$-carboxylate protrudes out of the cavity.

\section{Glycogen Phosphorylase}

Glycogen phosphorylase is involved in glycogen metabolism that regulates blood glucose levels and is an important therapeutic target for diabetes. This enzyme catalyses the phosphorylitic cleavage of glycogen to glycogen phosphate. This enzymatic reaction uses pyridoxal phosphate (PLP), a derivative of vitamin 6. Methotrexate, $3^{\prime}$-chloro- and $3^{\prime}, 5^{\prime}$ dichloromethotrexates and various folate derivatives have been shown to be reversible inhibitors of muscle glycogen phosphorylase $b$ (45). In this case, it is easy for us to argue that glycogen phosphorylase is a direct binder of the MTX probe. Our docking experiments on the structure of glycogen phosphorylase (1GGN) (46) also corroborates this hypothesis, as MTX in several of the docking poses is found with the $\gamma$-carboxylate protruding out of the cavity. 


\section{Deoxyguanosine Kinase and Deoxycytidine Kinase}

These enzymes are members of the deoxyribonucleoside kinases that phosphorylate deoxyribonucleosides, a crucial reaction in biosynthesis of DNA precursors through the salvage pathway. These kinases are of therapeutic interest as they are crucial in the activation of a number of anticancer and antiviral pro-drugs such as 2-chloro-2'-deoxyadenosine, azidothymidine and acyclovir (47). The crystal structure of deoxycytidine kinase has been recently solved (1P62) (48). Docking into the substrate binding site of deoxycytidine kinase produced binding modes where the complete MTX molecule bound as a bisubstrate, by blocking both substrate and ATP binding site. In all cases the $\gamma$-carboxylate points towards the entrance to the ATP binding site overlapping with the adenine ring. These binding modes are consistent with direct binding. This is consistent with observations that treatment with MTX increases the concentration of deoxycytidine triphosphate pools in L1210 ascites cells (49). It has already been suggested that MTX could be potentiating this effect by allosteric regulation of deoxycytidine kinase. Our results indicate that direct inhibition of deoxycytidine kinase is the most likely cause of the increased deoxycytidine triphosphate pools.

Likewise the structure of deoxyguanosine kinase (1JAG) $(50,51)$ is available. Interestingly, the crystal structure of deoxyguanosine kinase in complex with ATP showed the ATP molecule sitting in the substrate binding site rather than in the expected ATP binding site. This indicates that this subsite is quite flexible in accommodating molecules with differing chemotypes. Docking MTX in the site resulted in structures quite similar to those observed in the structurally conserved deoxycytidine kinase. This further corroborates that these two kinases are likely being isolated in our experiments through direct interactions with our MTX affinity probe.

\section{Pyridoxal Kinase}

We identified another PLP requiring enzyme, pyridoxal kinase. This enzyme catalyzes the conversion of pyridoxal to pyridoxal-5'-phosphate (PLP). PLP is an important cofactor in a variety of reactions such as decarboxylations, deaminations, transaminations, racemizations and aldol cleavages. Pyridoxal kinase shows up consistently as an intense band on our gels. The crystal structure of pyridoxal kinase was recently solved (1LHR) (52) in complex with the ATP analog AMP-PNP. Docking in the ATP binding site of pyridoxal kinase generated several binding poses suggestive of direct binding. In these poses the MTX overlaps well over the position of AMP-PNP and the pterin group makes similar hydrogen bonding contacts to the backbone atoms in the hinge region. The $\gamma$-carboxylate points towards the solvent exposed region on the kinase active site or cleft. It is our view that pyridoxal kinase is another direct binder of MTX. This is consistent with observations that alkylxanthines are ATP competitive inhibitors of pyridoxal kinase (53). As already argued earlier for HGPRT, the pterin group of MTX can act as a substitute of the xanthine moiety. Furthermore, extensive medicinal chemistry work done on antimetabolite research has elucidated that the pterin ring can be replaced with xanthine and xanthinelike moieties. Examples of this are Pemetrexed, (ALIMTA, LY-231514) the classical antimetabolite TS inhibitor drug from Lilly (54) and Tomudex (ZD9331) the nonclassical TS inhibitor from AstraZeneca (55). Furthermore, the fact that another PLP dependent enzyme, glycogen phosphorylase, binds MTX further corroborates that pyridoxal kinase is most likely binding through a direct interaction with the tethered MTX molecule. 


\section{Nudix I and 5}

Nudix hydrolases are housekeeping proteins involved in the hydrolysis of nucleoside phosphates. Nudix-1 (MTH1) for example, hydrolyses 8-oxo-dGTP and thus avoids errors caused by their misincorporation during DNA replication or transcription, which may result in carcinogenesis or neurodegeneration (56). Nudix 5 hydrolyses ADP sugars to AMP and sugar-5-phosphates. Nudix hydrolases that degrade dinucleosides and diphosphoinositol polyphosphates also have 5-phosphoribosyl 1-pyrophosphate (PRPP) pyrophosphatase activity that generates the glycolytic activator ribose 1,5-bisphosphate (57). The fact that these enzymes bind nucleotides and PRPP, two substrates already encountered in several other of the targets believed to be direct interactors of MTX, and their role in purine and pyrimidine synthesis is significant. Several crystal structures examples for ADP nudix hydrolases are available in the protein databank, but none that represent 8-oxo-dGTP hydrolase (58). We obtained the crystal structure of an ADP nudix hydrolases (nudix 5, 1KHZ) (59) and docked MTX into the nucleotide binding site. Interestingly, poses of MTX were found that are consistent with a direct interaction. The glutamate group can protrude out of the cavity, while the aminopterin group is buried well within the binding site making strong hydrogen bonding interactions. Although there is no evidence in the literature that nudix hydrolases bind folates or MTX, we believe that the presence of these proteins (at least nudix 5) in our gels is resulting from direct interactions with the MTX probe.

\section{Proteins Not Directly Associated With MTX}

The following proteins were captured in our experiments, but visual inspection of their crystal structure, inverse-docking and literature searches all indicate that their presence in our gels were likely a consequence of indirect binding with a direct MTX interactor. In particular, several of these were part of multifunctional proteins of the de novo purine synthetic pathway that are likely identified due to indirect interactions with other MTX binding proteins. For example AIRS and GARS are part of a trifunctional enzyme, which contains the direct interactor GART domain.

\section{Aminoimidazoleribonucleotide (AIR) Carboxylase}

AIR carboxylase catalyses the carboxylation of aminoimidazoleribonucleotide (60). The domain associated with this enzymatic activity in animals is part of a bifunctional polypeptide containing SAICAR synthase and AIR carboxylase. In our experiments a single band contained peptides from both domains of the bifunctional enzyme. The crystal structure of Air carboxylase (1D7A) (61) is available from the protein databank in complex with AIR. Docking runs of MTX in the air binding-site resulted in several poses that could also be compatible with binding. In these poses the pterin moiety of MTX is perpendicular to the imidazole ring of Air, but the $\gamma$-carboxylate does protrude out of the cavity. Because of this perpendicular binding, it is hard to postulate that this is a direct binder. We believe, however, that AIR carboxylase was captured due to protein-protein association with GARSAIRS-GART through the GART domain.

\section{Phosphoribosylaminoimidazolesuccino- carboxamide (SAICAR) Synthase}

This enzyme catalyses the seventh step in the de novo biosynthesis of purine nucleotides. The crystal structure of SAICAR synthase (1A48) (62) reveals that its active site is a very open cleft. There is no precedence in the literature for direct binding of SAICAR to folates 
or MTX. Docking experiments resulted only in poses in which the complete MTX molecule is buried deep into the cleft. In all poses both carboxylate groups are involved in hydrogen bonding interactions and fully buried inside the protein and would therefore interfere with binding to the attached MTX.

\section{Glycinamideribonucleotide Synthase (GARS)}

As mentioned earlier, the second, third, and fifth steps of de novo purine biosynthesis are catalyzed by a trifunctional protein GARSAIRS-GART (63). GARS catalyses the second step of the de novo purine biosynthetic pathway, the conversion of phosphoribosylamine, glycine, and ATP to glycinamide ribonucleotide (GAR), ADP, and Pi. We isolated peptides for GARS as part of the trifunctional protein GARS-AIRS-GART, but also as a separate band of $50 \mathrm{kDa}$ in the gel. Transfection of chinese hamster ovaries $(\mathrm{CHO})$ cell with the human GARS-AIRS-GART gene, has shown that this gene encodes not only the trifunctional protein of $110 \mathrm{kDa}$, but also a monofunctional GARS protein of $50 \mathrm{kDa}$ produced by alternative splicing resulting in the use of a polyadenylation site in the intron between the terminal GARS and the first AIRS exons (64). The mechanism of MTX binding was also investigated by docking experiments on the crystal structure of GARS (1GSO) (65). This protein, like SAICAR synthase has a very large open binding site, and no docking conformations were found where MTX could form productive stable complex with GARS. Since we found GARS as a separate band and docking experiments were inconclusive, it is difficult to postulate a direct binding mechanism. Eben though GART and GARS are part of the same trifunctional protein, there may be a protein-protein docking interaction between the domains. Protein-protein interactions between the first and second enzymes in purine biosynthesis, amido phosphoribosyl- transferase, and GARS, have also been postulated. Phosphoribosylamine is the product of the first enzyme and the substrate for the next reaction in the purine biosynthesis chain of events. There is evidence that this phosphoribosylamine reagent transfer occurs from one enzyme to the next via a coupling between Amidophosphoribosyltransferase and GARS, rather than through free diffusion (66). This presents a second possible mechanism for the association of GARS with MTX, and it is unlikely this enzyme interacts directly with the MTX probe.

\section{Phosphoribosylaminoimidazole Synthetase (AIRS)}

This enzyme is also part of the trifunctional, GARS-AIRS-GART protein. Docking runs on the crystal structure of AIRS (1CLI) (67) does not indicate direct binding with the MTX probe. We postulate that the presence of this enzyme is simply due to the fact that it is part of the trifunctional protein GARS-AIRSGART.

\section{Gluthathione Synthase}

Interestingly glutathione synthase is structural related to SAICAR synthase (68). Structural comparisons of these two proteins reveal a common fold. This fold is also shared with heat shock protein HSP70. HSP70 and HSP60 were also identified in these experiments, but since these proteins are commonly found in other experiments unrelated to the MTX probe, we excluded them from our discussion. However, because of the structural similarity with SAICAR synthase and Gluthathione synthase, it may be that these proteins are being pulled down by the same mechanism. The crystal structure of glutathione synthase is available (1GSA) (69) and was used in docking exercises that were also inconclusive. In all docking modes the complete MTX molecule is completely buried deep within a very closed active site pocket. 


\section{Propionyl CoA Carboxylase and Divalent Cation Tolerant Protein (CUTA)}

These proteins are pulled down consistently in our experiments. The crystal structure of proprionyl CoA carboxylase is not known and a literature search does not show previous evidence of any interaction between MTX and this enzyme. The crystal structure of CUTA was recently solved and is available (1P1L) (70) as part of a structural genomics initiative, however the function of this enzyme is yet not understood. Docking of MTX in this crystal structure was inconclusive.

\section{Known Folate Binders Not Identified}

As discussed earlier, folates and antifolates, such as MTX are known to bind or be processed by several folate-binding proteins not captured in our experiments. To our surprise, our experiments failed to isolate AICARFT, SHMT, FPGS, $\gamma-\mathrm{GH}$, and RFC. Inspection of their corresponding crystal structures and knowledge of the mechanism of catalysis of these enzymes allowed us to rationalize the reason for their absence. Inspection of the recently solved crystal structure of AICARFT with a multisubstrate folate inhibitor (1OZ0) (71) indicates that while the $\gamma$-carboxylate of the molecule points out of the cavity, it does not protrude far enough to preclude possible steric interaction of surrounding protein sidechains with the MTX matrix. Furthermore, it is known that MTX is only a weak inhibitor of AICARFT as compared to other targets. The case for FPGS (1JBW) (72) and $\gamma$-GH (1L9X) (73) is easier to make as the crystal structures of these two proteins have been solved and their mechanism of catalysis are now well understood. The glutamate sidechain of MTX and folates needs to point into the cavity in order for polymerization or hydrolysis to occur, a condition incompatible with binding our MTX affinity probe. The crystal structure of Serine Hydroxymethyltransferase in com- plex with glycine and 5-formyl tetrahydrofolate is also available (1DFO) (74). In this structure the pteridine ring is buried in the active site, but the $\gamma$-carboxylate protrudes well out of the cavity in a way that would be expected to bind the MTX probe. However, it is well known that the folate-independent cleavage of L-serine substrates is competitively inhibited by 5-formyl-H4PteGlu(n), but with dependence on the length $(n)$ of the polyglutamate chain. For monoglutamates inhibition is immediate, but for $n=3$ inhibition is very slow (75). We believe that the matrix in our MTX probe may be mimicking a long polyglutamate chain and that the time scale of our experiments is not long enough to observe binding. In the case of the membraneimbedded reduced folate carrier there is no crystal structure available and the mechanism of folate transport is not completely clear (76). We adopted protocols (not shown) specifically for isolating proteins from membrane fractions but persistently failed to isolate this protein.

\section{The Purine Metabolone: Proposal of Enzyme Complexes for Substrate Channeling}

The concept of substrate channeling (77), or rather the transfer of metabolites between consecutive enzymes in a reaction pathway is a topic of much controversy. While inter-domain channeling within a single enzyme, as seen in the ammonia transfer between the glutaminase and the phosphoribosyltransferase active sites in amido phosphoribosyltransferase, is well established as a result of recent crystallographic evidence for the formation of a hydrophobic channel connecting the two active sites (78), channeling between two consecutive enzymes in a reaction pathway is less understood. Part of the controversy of inter-enzyme channeling arises from the fact that within systems reported to be involved in channeling, interacting enzymes do not form stable complexes. It has been proposed that in these systems the 
channeling is a dynamic process involving an encounter between the enzyme-substrate complex and the next enzyme occurring at a faster rate than the process of free diffusion of substrate from the first enzyme into the medium followed by complex formation with the second enzyme (79). Although there has been no direct evidence for formation of a complex between amido phosphoribosyltransferase and GARS under reaction conditions, a channeling mechanism has been proposed for the transfer of phosphoribosylamine (PRA) from amido phosphoribosyltransferase to GARS (80). PRA is highly unstable under physiological conditions and therefore needs to be protected from the medium via the formation of a transient channel between these two enzymes so that it can be quickly transformed into the stable intermediate GAR by GARS. We propose that the identification of a least three proteins containing enzymatic activity for six steps in the de novo purine synthesis pathway may represent evidence for a pathway architecture that is consistent with substrate channeling $(77,81)$, given that only one is known to be inhibited by antifolate metabolites such as MTX. Already substrate channeling has been proposed for GARS and amido phosphoribosyltransferase. GARS is part of a trifunctional protein also containing activities for AIRS and GART respectively. This trifunctional protein catalyzes the second, third, and fifth step in de novo synthesis. There is evidence for GART interaction with MTX, but not for GARS and AIRS. We did not find evidence in our experiments for the presence of the fourth enzyme in the pathway, FGARAT, but we did find the bifunctional protein containing activities for AIR carboxylase and SAICAR synthase, that catalyze the sixth and seventh steps in the pathway. We could not find evidence in the literature for direct inhibition of either AIR carboxylase or SAICAR synthase by MTX or folate analogs. Furthermore, our docking experiments suggest that this protein was isolated because of secondary interactions with a MTX binding protein as a binding mode indicating direct binding was not found. We believe that this protein is specifically interacting with GARS-GARTAIRS, likely via interactions through the AIRS domain, which comes a step before AIR carboxylase in the sequence. We propose that enzymes bound to MTX directly are likely to represent inhibited states whose structures may be are more compatible with engaging other enzymes in the pathway via stable proteinprotein interactions.

We did not identify succino adenyl synthase, the eighth enzyme in the pathway or the bifunctional protein containing activities for the folate-dependent enzyme AICARFT and IMP cyclohydrolase, that catalyze the ninth step and the tenth step in de novo purine synthesis, respectively. It is known, however, that MTX is a weaker inhibitor of AICARFT than other folate dependent enzymes in the pathway. Examination of the crystal structure of AICARFT with a folate analog suggest that the position of the $\gamma$-carboxylate may be too buried in the enzyme to preclude MTX binding because of possible steric interactions with the matrix.

The functions of the enzymes of de novo purine synthesis pathway have been well studied, however, the underlying structural organization of these enzymes is less well understood. It is reasonable to expect that a high degree of scaffolding is engineered into these structures that is consistent with substrate channeling from one enzyme to another in order to protect unstable reactive intermediates from solvent and for reaction efficiency. It has also been proposed that substrate channeling helps direct the flux of a pathway by eliminating the competition from other enzymes for common metabolic intermediates. It is conceivable that MTX as an inhibitor may stabilize the structure of those enzymes it 
binds resulting in conformations more conducive to stable complex formation with other enzymes in the pathway. By identifying such a large portion of this pathway we present evidence that the enzymes of the purine biosynthesis pathway are scaffolded together in a manner consistent with substrate channeling.

\section{Conclusions}

Methotrexate is an important drug with applications in several therapeutic areas with unmet medical needs. The efficacy of this drug in many cases has been arrived at serendipitously. Although it has been widely used in rheumatoid arthritis and immunosuppression a clear mechanism of action is not yet available. We were able to identify the three main therapeutic targets of antifolate therapies in the clinic in a single experiment. It has been postulated that MTX is able to interact with at least eight other proteins not widely regarded as targets of this drug but with crucial roles in medicine and drug discovery. Inhibition of IMPDH by MTX, for example, may be the underlying reason behind its efficacy as an immunosuppressive agent, and inhibition of the first enzyme in the de novo synthesis of nucleotides, amidophosphoribosyltransferase may be responsible at least in part for its efficacy in Rheumatoid arthritis.

Another aspect we believe has paramount importance is the capture in a single experiment of such a large portion of the de novo and salvage nucleotide synthesis pathways. Six of the ten steps in purine synthesis are carried out by enzymes identified with our drug probe. This remarkable finding indicates that these proteins, like signal transduction proteins, are structurally engineered in such a way as to facilitate the transfer of the evolving reagent (purine) from one enzyme to the next via tandem protein-protein recognition events. Furthermore, the fact that so many of the proteins identified in these experiment represent viable drug discovery targets in the pharmaceutical industry underlines the effectiveness of this technique in target discovery. The application of this approach to other drugs and drug candidates may facilitate the prediction of unknown and secondary therapeutic target proteins and those related to the side effects and toxicity. These results demonstrate that our proteomics technology could play an important role in drug discovery since it allows monitoring of the interactions between a drug and the protein content of a cell with affinities over a very large dynamic range as indicated by identifying DHFR (Kd approx $10 \mathrm{nM}$ ) and TS (Kd approx $25 \mu M$ ). This technology has particular promise as a tool to stratify patient populations for clinical studies by developing drug protein fingerprints that can be correlated with patient compliance. Drug response is a very complex event. A drug's proteomics fingerprint represents a Pharmaco-dynamic/Pharmaco-kinetic filter that allows only relevant proteins to be monitored. By monitoring a full compliment of proteins that interact with a drug the underlying reason for response may be revealed.

We would like to acknowledge Robert Rottapel, Daniel Figeys, David Stover, Henry Duewel, Olga Ornatsky, and Irving Sucholeiki for helpful discussions during this work.

\section{References}

1. Arlington SA: Industrialization of $R \& D$ in the 21st century. ECPI-Barcelona 2001, PricewatersCoopers

2. CHI, Pharmacogenomics/Pharmacoproteomics, Europe. May 2002, Munich, Germany.

3. Leung D, Hardouin C, Boger DL, Cravatt BF. Discovering potent and selective reversible inhibitors of enzymes in complex proteomes. Nat Biotechnol. 2003;21(6):687-691.

4. Kim E, Park JM. Identification of Novel Target Proteins of Cyclic GMP Signaling Pathways Using Chemical Proteomics. J Biochem Mol Biol. 2003;36(3):299-304.

5. Graves PR, Kwiek JJ, Fadden P, et al. Discovery of novel targets of quinoline drugs in the 
human purine binding proteome. Mol Pharmacol. 2002;62(6):1364-1372.

6. Ho Y, Gruhler A, Heilbut A, et al. Systematic identification of protein complexes in Saccharomyces cerevisiae by mass spectrometry. Nature 2002;415(6868):180-183

7. Chen YZ, Zhi DG. Ligand-protein inverse docking and its potential use in the computer search of protein targets of a small molecule. Proteins 2001;43(2):217-226.

8. Chen YZ, Ung CY. Prediction of potential toxicity and side effect protein targets of a small molecule by a ligand-protein inverse docking approach. J Mol Graph Model 2001;20(3): 199-218.

9. Smith-Schmidt T. Banking on Structures. BioIT World 2002:1 (8).

10. Clark RD, Strizhev A, Leonard JM, Blake JF, Matthew JB. Consensus scoring for ligand/ protein interactions. J Mol Graph Model. 2002; 20(4):281-295.

11. Saravanan V, Hamilton J. Advances in the treatment of rheumatoid arthritis: old versus new therapies. Expert Opin Pharmacother 2002;3(7):845-856.

12. Allison AC. Immunosuppressive drugs: the first 50 years and a glance forward. Immunopharmacology 2000;47(2-3):63-83.

13. Weber G, Prajda N. Targeted and non-targeted actions of anti-cancer drugs. Adv Enzyme Regul 1994;34:71-89.

14. Cronstein $\mathrm{BN}$. The mechanism of action of methotrexate. Rheum Dis Clin North Am 1997; 23(4):739-755.

15. Costi MP, Ferrari S. Update on antifolate drugs targets. Curr Drug Targets. 2001;2(2): 135-166.

16. Kaye SB. New antimetabolites in cancer chemotherapy and their clinical impact. Br J Cancer 1998;78 Suppl 3:1-7.

17. Allegra CJ, Drake JC, Jolivet J, Chabner BA. Inhibition of phosphoribosylaminoimidazolecarboxamide transformylase by methotrexate and dihydrofolic acid polyglutamates. Proc Natl Acad Sci USA 1985;82(15):4881-4885.

18. Prabhu V, Chatson KB, Lui H, Abrams GD, King J. Effects of sulfanilamide and methotrexate on $13 \mathrm{C}$ fluxes through the glycine decarboxylase/serine hydroxymethyltransferase enzyme system in arabidopsis. Plant Physiol 1998;116:137-144.
19. Aghi M, Kramm CM, Breakefield XO. Folylpolyglutamyl synthetase gene transfer and glioma antifolate sensitivity in culture and in vivo. J Natl Cancer Inst 1999;91(14):1233-1241.

20. Cole PD, Kamen BA, Gorlick R, et al. Effects of overexpression of $\gamma$-Glutamyl hydrolase on methotrexate metabolism and resistance. Cancer Res 2001;61(11):4599-4604

21. Sierra EE, Goldman ID. Recent advances in the understanding of the mechanism of membrane transport of folates and antifolates. Semin Oncol 1999;26(2 Suppl 6):11-23.

22. Mauritz R, Peters GJ, Priest DG, et al. Multiple mechanisms of resistance to methotrexate and novel antifolates in human CCRF-CEM leukemia cells and their implications for folate homeostasis. Biochem Pharmacol 2002;63(2): 105-115.

23. Sawaya MR, Kraut J. Loop and subdomain movements in the mechanism of Escherichia coli dihydrofolate reductase: crystallographic evidence. Biochemistry 1997 Jan 21; 36(3):586-603

24. Fritz TA, Tondi D, Finer-Moore JS, Costi MP, Stroud RM., Predicting and harnessing protein flexibility in the design of species-specific inhibitors of thymidylate synthase. Chem Biol 2001;8(10):981-995.

25. Almassy RJ, Janson CA, Kan CC, Hostomska Z. Structures of apo and complexed Escherichia coli glycinamide ribonucleotide transformylase. Proc Natl Acad Sci USA 1992; 89(13):6114-6118.

26. Rajagopalan PT, Zhang Z, McCourt L, Dwyer M, Benkovic SJ, Hammes GG. Interaction of dihydrofolate reductase with methotrexate: ensemble and single-molecule kinetics. Proc Natl Acad Sci U S A. 2002;99(21):13481-13486.

27. Gangjee A, Yu J, McGuire JJ, Cody V, Galitsky N, Kisliuk RL, Queener SF. Design, synthesis, and X-ray crystal structure of a potent dual inhibitor of thymidylate synthase and dihydrofolate reductase as an antitumor agent. J Med Chem 2000;43(21):3837-3851.

28. Galivan JH, Maley GF, Maley F. Factors affecting substrate binding in Lactobacillus casei thymidylate synthetase as studied by equilibrium dialysis. Biochemistry. 1976; 15(2):356-362.

29. Smith JL. Glutamine PRPP amidotransferase: snapshots of an enzyme in action. Curr Opin Struct Biol 1998;8(6):686-694. 
30. Chen S, Tomchick DR, Wolle D, et al. Mechanism of the synergistic end-product regulation of Bacillus subtilis glutamine phosphoribosylpyrophosphate amidotransferase by nucleotides. Biochemistry 1997;36(35):10718-10726.

31. Sant ME, Lyons SD, Phillips L, Christopherson RI. Antifolates induce inhibition of amido phosphoribosyltransferase in leukemia cells. J Biol Chem 1992;267(16):11038-11045.

32. Schoettle SL, Christopherson RI. Inhibition of murine amido phosphoribosyltransferase by folate derivatives. Adv Exp Med Biol 1994; 370:151-154.

33. Genestier L, Paillot R, Fournel S, Ferraro C, Miossec P, Revillard JP. Immunosuppressive properties of methotrexate: apoptosis and clonal deletion of activated peripheral T cells. J Clin Invest 1998;102(2):322-328.

34. Fairbanks LD, Ruckemann K, Qiu Y, Hawrylowicz CM, Richards DF, Swaminathan R, Kirschbaum B, Simmonds HA. Methotrexate inhibits the first committed step of purine biosynthesis in mitogen-stimulated human T-lymphocytes: a metabolic basis for efficacy in rheumatoid arthritis? Biochem J 1999;342 (Pt 1):143-152.

35. Jain J, Almquist SJ, Shlyakhter D, Harding MW. VX-497: a novel, selective IMPDH inhibitor and immunosuppressive agent. J Pharm Sci 2001;90(5):625-637.

36. Prosise GL, Luecke H. Crystal structures of Tritrichomonasfoetus inosine monophosphate dehydrogenase in complex with substrate, cofactor and analogs: a structural basis for the random-in ordered-out kinetic mechanism. J Mol Biol. 2003;326(2):517-527

37. Sintchak MD, Fleming MA, Futer O, Raybuck SA, Chambers SP, Caron PR, Murcko MA, Wilson KP. Structure and mechanism of inosine monophosphate dehydrogenase in complex with the immunosuppressant mycophenolic acid. Cell. 1996;85(6):921-930.

38. Weber G, Nagai M, Natsumeda Y, Ichikawa S, et al. Regulation of de novo and salvage pathways in chemotherapy. Adv Enzyme Regul 1991;31:45-67.

39. Weber G, Prajda N. Targeted and non-targeted actions of anti-cancer drugs. Adv Enzyme Regul 1994;34:71-89.

40. Gordon RB, Keough DT, Emmerson BT. HPRTdeficiency associated with normal PRPP con- centration and APRT activity. J Inherit Metab Dis 1987;10(1):82-88.

41. Fung KP, Lam WP, Choy YM, Lee CY. Effect of methotrexate on the intracellular phosphoribosyl pyrophosphate level and glucose transport of Ehrlich ascites tumor cells in vitro. Oncology 1996;53(1):27-30.

42. Balendiran GK, Molina JA, Xu Y, et al. Ternary complex structure of human HGPRTase, PRPP, $\mathrm{Mg} 2+$, and the inhibitor HPP reveals the involvement of the flexible loop in substrate binding. Protein Sci 1999;8(5):1023-1031.

43. van Ede AE, Laan RF, Blom HJ, et al. Homocysteine and folate status in methotrexatetreated patients with rheumatoid arthritis. Rheumatology (Oxford) 2002;41(6):658-665.

44. Cronk JD, Endrizzi JA, Alber T. Highresolution structures of the bifunctional enzyme and transcriptional coactivator $\mathrm{DCoH}$ and its complex with a product analogue. Protein Sci 1996;5(10):1963-1972.

45. Klinov SV, Chebotareva NA, Sheiman BM, Birinberg EM, Kurganov BI. Interaction of muscle glycogen phosphorylase B with methotrexate, folic and folinic acids. Bioorg Khim 1987;13(7):908-914.

46. Zographos SE, Oikonomakos NG, Tsitsanou $\mathrm{KE}$, et al. The structure of glycogen phosphorylase B with an alkyldihydropyridinedicarboxylic acid compound, a novel and potent inhibitor. Structure 1997;5(11):1413-1125.

47. Johansson NG, Eriksson S. Structure-activity relationships for phosphorylation of nucleoside analogs to monophosphates by nucleoside kinases. Acta Biochim Pol 1996;43(1):143-160.

48. Sabini E, Ort S, Monnerjahn C, Konrad M, Lavie A. Structure of human dCK suggests strategies to improve anticancer and antiviral therapy. Nat Struct Biol. 2003;10(7): 513-519.

49. Roberts D, Peck C. Effect of methotrexate and $1-\beta$-D-arabinofuranosylcytosine on pools of deoxyribonucleoside triphosphates in L1210 ascites cells. Cancer Res. 1981;41(2):505-510.

50. Mikkelsen NE, Johansson K, Karlsson A, Knecht W, Andersen G, Piskur J, MunchPetersen B, Eklund H. Structural basis for feedback inhibition of the deoxyribonucleoside salvage pathway: studies of the Drosophila deoxyribonucleoside kinase. Biochemistry. 2003;42(19):5706-5712. 
51. Johansson K, Ramaswamy S, Ljungcrantz C, Knecht W, Piskur J, Munch-Petersen B, Eriksson S, Eklund H. Structural basis for substrate specificities of cellular deoxyribonucleoside kinases. Nat Struct Biol. 2001;8(7):616-620.

52. Li MH, Kowk F, Chang WR, et al. Crystal structure of brain pyridoxal kinase, a novel member of the ribokinase superfamily. J Biol Chem 2002;277(48):46385-46390.

53. Ubbink JB, Bissbort S, Vermaak WJ, Delport R. Inhibition of pyridoxal kinase by methylxanthines. Enzyme 1990;43(2):72-79.

54. Jones RJ, Twelves CJ. Pemetrexed: a multitargeted antifolate (ALIMTA, LY-231514). Expert Rev Anticancer Ther 2002;2(1):13-22.

55. Aherne GW, Hardcastle A, Ward E, Dobinson D, Crompton T, Valenti M, Brunton L, Jackman AL. Pharmacokinetic/pharmacodynamic study of ZD9331, a nonpolyglutamatable inhibitor of thymidylate synthase, in a murine model following two curative administration schedules. Clin Cancer Res 2001;7(9):2923-2930.

56. Sakai Y, Furuichi M, Takahashi M, Mishima M, Iwai S, Shirakawa M, Nakabeppu Y. A molecular basis for the selective recognition of 2-hydroxy-dATP and 8-oxo-dGTP by human MTH1. J Biol Chem 2002; 277(10): 8579-8587.

57. Fisher DL, Safrany ST, McLennan AG, Cartwright JL. Nudix hydrolases that degrade dinucleoside and diphosphoinositol polyphosphates also have 5-phosphoribosyl 1-pyrophosphate (PRPP) pyrophosphatase activity that generates the glycolytic activator ribose 1,5-bisphosphate. J Biol Chem 2002;277(49):47313-47317.

58. Gabelli SB, Bianchet MA, Bessman MJ, Amzel LM. The structure of ADP-ribose pyrophosphatase reveals the structural basis for the versatility of the Nudix family. Nat Struct Biol 2001;8(5):467-472.

59. Gabelli SB, Bianchet MA, Ohnishi Y, Ichikawa Y, Bessman MJ, Amzel LM. Mechanism of the Escherichia coli ADP-ribose pyrophosphatase, a Nudix hydrolase. Biochemistry 2002;41: 9279-9285.

60. Chen ZD, Dixon JE, Zalkin H. Cloning of a chicken liver cDNA encoding 5-aminoimidazole ribonucleotide carboxylase and 5-aminoimidazole-4-N-succinocarboxamide ribonucleotide synthetase by functional complementation of Escherichia coli pur mutants. Proc Natl Acad Sci USA 1990;87(8):3097-101.
61. Mathews II, Kappock TJ, Stubbe J, Ealick SE. Crystal structure of Escherichia coli PurE, an unusual mutase in the purine biosynthetic pathway. Structure Fold Des 1999;7(11): 1395-1406.

62. Levdikov VM, Barynin VV, Grebenko AI, Melik-Adamyan WR, Lamzin VS, Wilson KS. The structure of SAICAR synthase: an enzyme in the de novo pathway of purine nucleotide biosynthesis. Structure 1998;6(3):363-376.

63. Kan JL, Moran RG. Analysis of a mouse gene encoding three steps of purine synthesis reveals use of an intronic polyadenylation signal without alternative exon usage. J Biol Chem 1995;270(4):1823-1832.

64. Brodsky G, Barnes T, Bleskan J, Becker L, Cox M, Patterson D. The human GARS-AIRS-GART gene encodes two proteins which are differentially expressed during human brain development and temporally overexpressed in cerebellum of individuals with Down syndrome. Hum Mol Genet 1997;6(12):2043-2050.

65. Wang W, Kappock TJ, Stubbe J, Ealick SE. $\mathrm{X}$-ray crystal structure of glycinamide ribonucleotide synthetase from Escherichia coli. Biochemistry 1998;37(45):15647-15662.

66. Bera AK, Chen S, Smith JL, Zalkin H. Temperature-dependent function of the glutamine phosphoribosylpyrophosphate amidotransferase ammonia channel and coupling with glycinamide ribonucleotide synthetase in a hyperthermophile. J Bacteriol 2000;182(13): 3734-3739.

67. Li C, Kappock TJ, Stubbe J, Weaver TM, Ealick SE. X-ray crystal structure of aminoimidazole ribonucleotide synthetase (PurM), from the Escherichia coli purine biosynthetic pathway at 2.5 A resolution. Structure Fold Des 1999; 7(9): 1155-1166.

68. Levdikov VM, Barynin VV, Grebenko AI, Melik-Adamyan WR, Lamzin VS, Wilson KS. The structure of SAICAR synthase: an enzyme in the de novo pathway of purine nucleotide biosynthesis. Structure 1998;6(3):363-376.

69. Hara T, Kato H, Katsube Y, Oda J. A pseudomichaelis quaternary complex in the reverse reaction of a ligase: structure of Escherichia coli B glutathione synthetase complexed with ADP, glutathione, and sulfate at 2.0 A resolution. Biochemistry 1996;35(37):11967-11974.

70. Kniewel R, Buglino JA, Lima CD. Structure of the Periplasmic Divalent Cation Tolerance 
Protein Cuta From Archaeoglobus Fulgidus. 2003; in press.

71. Wolan DW, Greasley SE, Wall MJ, Benkovic SJ, Wilson IA. Structore of avian AICAR transformylase with a multisubstrate adduct inhibitor beta-DADF identifies the folate binding site. Biochemistry 2003;42(37):10904-10914.

72. Sun $X$, Cross JA, Bognar AL, Baker EN, Smith CA. Folate-binding triggers the activation of folylpolygutamate synthetase. J Mol Biol 2001;310(5):1067-1078.

73. Li H, Ryan TJ, Chave KJ, Van Roey P. ThreeDimensional Structure of Human GammaGlutamyl Hydrolase. A Class I Glutamine Amidotransferase Adapted for a Complex Substrate. J Bio Chem 2002;277(27):24522-24529.

74. Scarsdale JN, Radaev S, Kazanina G, Schirch V, Wright HT. Crystal structure at 2.4 A resolution of $E$. coli serine hydroxymethyltransferase in complex with glycine substrate and 5-formyl tetrahydrofolate. J Mol Biol 2002;296(1):155-168.

75. Stover P, Schirch V. 5-Formyltetrahydrofolate polyglutamates are slow tight binding inhibitors of serine hydroxymethyltransferase. J Biol Chem 1991;266(3):1543-1350.

76. Sierra EE, Goldman ID. Recent advances in the understanding of the mechanism of membrane transport of folates and antifolates. Semin Oncol 1999;262(2 Suppl 6): 11-23.

77. Perham RN. Swinging arms and swining domains in multifunctional enzymes: catalytic machines for multistep reactions. Annu Rev Biochem 2000;69:961-1004.

78. Krahn JM, Kim JH, Burns MR, Parry RJ, Zalkin H, Smith JL. Coupled formation of an amidotransferase interdomain ammonia channel and a phosphoribosyltransferase active site. Biochemistry 1997;36(37):11061-11068.

79. Haggie PM, Verkman AS. Diffusion of tricarboxylic acid cycle enzymes in the mitochrondrial matrix in vivo. Evidence for restricted mobility of a multienzyme complex. J Biol Chem 2002;277(43):40782-40788.

80. Bera AK, Chen S, Smith JL, Zalkin H. Temperature-dependent function of the glutamine phosphoribosylpyrophosphate amidotransferase ammonia channel and coupling with glycinamide ribonucleotide synthetase in a hyperthermophile. J Bacteriol 2000;182(13): 3734-3739.

81. Appling DR. Compartmentation of folatemediated one-carbon metabolism in eukaryotes. FASEB J 1991;5(12):2645-2651. 